Physics and Astrophysics of Planetary Systems

T. Montmerle, D. Ehrenreich and A.-M. Lagrange (eds)

EAS Publications Series, 41 (2010) 3-23

\title{
THE SEARCH FOR OTHER PLANETS AND LIFE
}

\author{
Ch. Beichman ${ }^{1}$
}

\begin{abstract}
This Les Houches School offers students a wide ranging view of the field of exoplanets and the search for life beyond the solar system. Observational and theoretical opportunities abound in a new field of astronomy that will be growing for decades to come. I give a brief introduction and overview to the many detailed talks that will be presented in this volume.
\end{abstract}

\section{Introduction}

\subsection{A Geographical Introduction: Science at Mont Blanc}

The buildings of the Les Houches school sit in the shadow of Mont Blanc in the French Alps. While not a modern astronomical site, Mont Blanc played an interesting role in the early development of modern astronomy. In 1864, the French scientist Jules Janssen went to the Alps to make measurements that would differentiate between solar and telluric lines in the spectra of the Sun just discovered by Bunsen and Kirchhoff. Janssen, who later served as the first Director of Meudon Observatory, succeeded in attributing some of the lines to terrestrial water vapor (Aubin 2007). Around 1890 he founded an observatory atop Mont Blanc which by then was an important outpost for observations in glaciology, astronomy, meteorology and medicine. One scientist died on Mont Blanc $(4350 \mathrm{~m})$ in that era from the first recorded high altitude pulmonary edema (Richalet 2001), an early warning for those of us who regularly go to high altitude observatories in Hawaii and Chile. In 1900, French graduate student Charles Nordman set up a long wire antenna on the Mont Blanc glacier to detect solar Hertzian waves (Nordman 1902). Had the Sun been at solar maximum, the honor of the first detection of extra-terrestrial radio waves would have belonged to France. Mont Blanc's role in science was at one time so well known that in 1930 the German film maker Arnold Franck made a thriller Storm Over Mont Blanc in which (according to Amazon.com), "a scientist

\footnotetext{
${ }^{1}$ Michelson Science Center, California Institute of Technology, 770 S. Wilson Ave, Pasadena, CA 91125, USA; e-mail: chas@ipac.caltech.edu
} 
works in courageous isolation an isolated observatory perched on the dangerous peak of Mont Blanc. He falls in love with a beautiful astronomer played by Leni Riefenstahl" - an actress who went on to a more turbulent and infamous career. And, of course, the Mont Blanc area has been host to the Les Houches Physics Schools for over 50 years.

\subsection{A Philosophical Introduction: The Many Worlds Hypothesis}

The questions of the existence of other worlds and of life beyond Earth goes back many centuries, at least to the pre-Socratic philosophers such as Epicurius who in 300 B.C.E posited that "There are infinite worlds both like and unlike this world of ours... We must believe that in all worlds there are living creatures and plants and other things we see in this world." In the Renaissance opinion was divided, particularly about the prospects of life, with Galileo stating flatly, "I (regard) as false and damnable the view of those who would put inhabitants on Jupiter, Venus, and Saturn, and the moon, meaning by inhabitants animals like ours and men in particular." Giordano Bruno, on the other hand, asserted, "There are countless suns and countless earths all rotating around their suns in exactly the same way as the seven planets of our system. We see only the suns because they are the largest bodies and are luminous, but their planets remain invisible to us because they are smaller and non-luminous. The countless worlds in the universe are no worse and no less inhabited than our Earth" (Bruno 1584). Natural Philosophers of the Enlightenment such as Christopher Huygens were well disposed to the existence of other worlds, other forms of plant and animal life, and even other "humans" who, he noted, are distinguished from beasts by their study of Nature, including "Astronomy and its subservient Arts: Geometry, Arithmetick, Writing, Opticks" (Huygens 1698).

As this workshop will demonstrate, our era is marked by rapid progress in our ability to detect and characterize other worlds with the tools of science, not philosophical deductions. But much speculation remains, as a multitude of science fiction books and movies can attest to. The boundary between science and speculation is perhaps best encompassed in the Drake Equation (as discussed in Drake \& Sobel 1992) which tries to quantify the number of inhabited worlds with which we might someday communicate:

$$
N=\mathbf{R}_{*} \times \mathbf{f}_{\mathbf{p}} \times \mathbf{n}_{\mathbf{e}} \times \mathbf{f}_{\mathbf{l}} \times f_{i} \times f_{c} \times L
$$

where $N$ is the number of communicative civilizations; $\mathbf{R}_{*}$ is the rate of formation of suitable stars; $\mathbf{f}_{p}$, the fraction of those stars with planets; $\mathbf{n}_{e}$, the number of Earth-like worlds per planetary system; $\mathbf{f}_{l}$, the fraction of those Earth-like planets where life actually develops; $f_{i}$, the fraction of life sites where intelligence develops; $f_{c}$, the fraction of communicative planets; and $L$, the "lifetime" of communicating civilizations. A decade ago we knew only the first term in the equation, the rate of star formation and nothing about the incidence of planets. As will be described below, we are right now engaged in measuring the "astronomical terms,"

$\mathbf{f}_{p}$ and $\mathbf{n}_{e}$. Within the next 25 years (or sooner, I hope), we will measure the first 


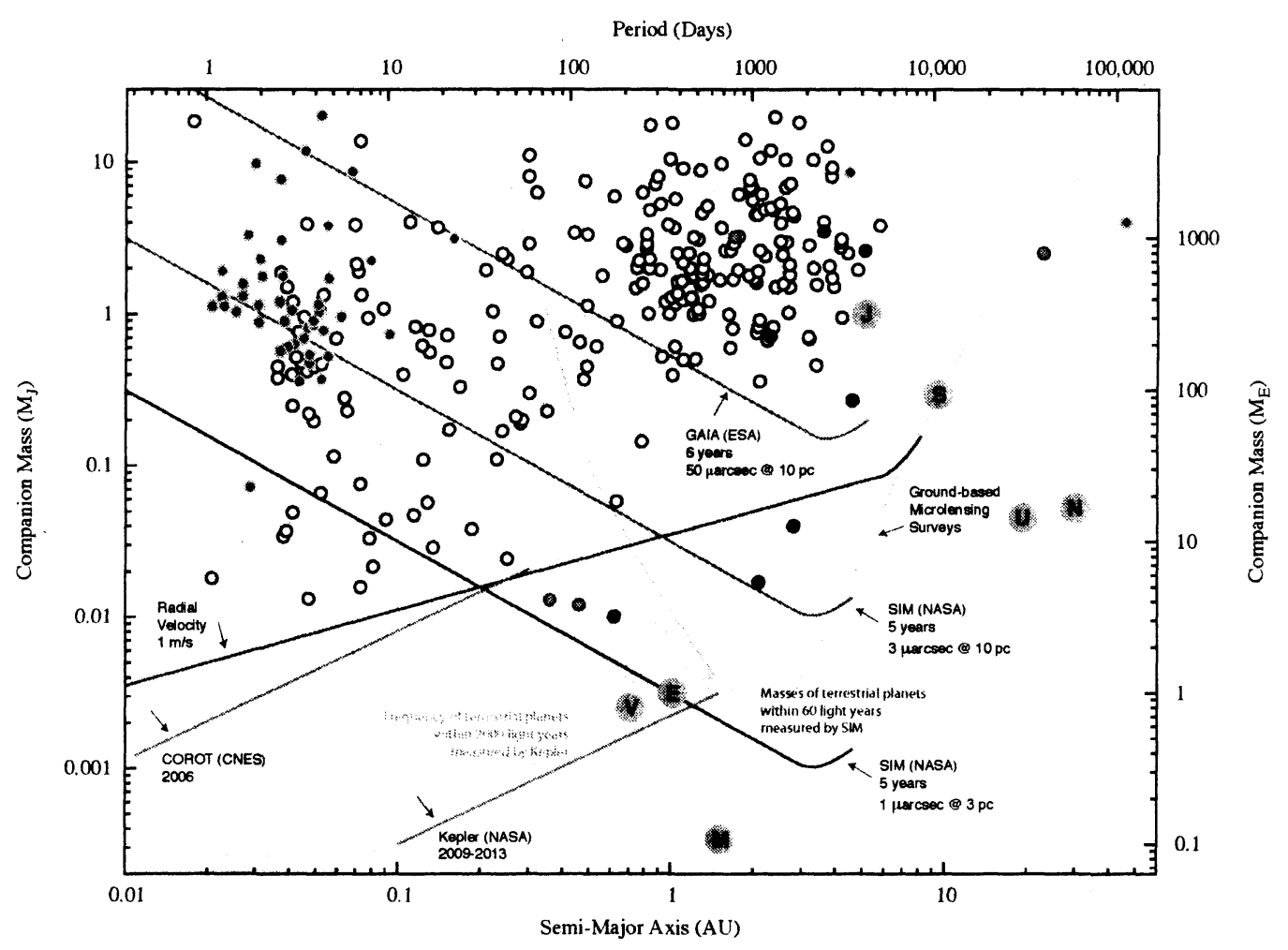

Fig. 1. Planets detected via various means are shown as circles: dark (blue) circles with light borders $(<0.1 \mathrm{AU}$ ) from transits; open circles from radial velocities; dark (red) with dark borders ( 1-5 AU) from microlensing; light (cyan) with dark borders from timing; and two dark (red) circles with light borders in the upper right from imaging. Sensitivity curves are given for radial velocity, astrometry and transits are labeled. Data from exoplanet encyclopedia (Schneider 2008). Figure courtesy of Peter Lawson (JPL).

"astrobiological term", $\mathbf{f}_{l}$, by examining the spectra of nearby worlds in search of habitable planets, with water and familiar atmospheric composition, and even look for evidence of primitive life. The remaining terms of the Drake Equation pertain to the evolution and survival of intelligent life (or at least of other electrical engineers) and will remain highly speculative until the success of one or more of the many SETI experiments (Tarter et al. 2007). But the astronomical investigation of $\mathbf{f}_{p}$ and $\mathbf{n}_{e}$ and the astrobiological investigation of $\mathbf{f}_{l}$ is a task of the highest scientific interest. The 2000 report of the US Decadal Survey called this "so challenging and of such importance that it could occupy astronomers for the foreseeable future." (McKee \& Taylor 2001).

\section{Indirect Techniques of Planet Detection}

The census of planets changes almost weekly, with roughly 30 planets being found per year through a variety of techniques. Figure 1 shows the mass and orbital location of many of the observed planets with lines showing sensitivity regimes 
for different techniques. This figure is a useful guide to the different techniques I will discuss. For more detailed discussions of these topics, please see the other talks in the volume. From Jean Schneider's invaluable exoplanet encyclopedia [http://exoplanet.eu] we find the following snapshot of the 309 planets detected as of July 2008:

- RadialVelocity: 221 planetary systems, 257 planets, 25 multiple systems.

- Transit: 52 planetary systems, 52 planets.

- Microlensing: 7 planets, 1 multiple system.

- Astrometry: 0 planets.

- Timing: 3 planetary systems, 5 planets, 1 multiple systems.

- Imaging: 5 planets.

A brief description of the present performance and long term potential of each technique is given in the following sections.

\subsection{Timing Measurements}

The first exo-planets detected were found not by the radial velocity technique around a solar type star, but by the timing of small shifts in the arrival times of pulses from a pulsar. The amplitude of the change in pulse arrival times is given approximately by:

$$
\text { Delay }(\mathrm{msec})=\frac{M_{p}}{M_{*}} \frac{D \sin i}{c} \sim 1.5 M_{\oplus} D_{A U}
$$

where $\left(M_{p} / M_{*}\right)$ is the planet-pulsar mass ratio, $D$ the orbital separation, $i$ the orbital inclination and $c$ the speed of light. Measurements of PSR B1257 +12 , a near-perfect clock with a $6.5 \mathrm{~m} \mathrm{~s}^{-1}$ period, revealed three planets with 25,66 and 98 day periods and masses of $0.02,4.3$ and $3.9 M_{\oplus}$, respectively (Wolczan \& Frail 1992; Konacki \& Wolczan 2003). The measurements are so precise that non-Keplerian interactions must be taken into account and demonstrate that the outer two planets are coplanar and locked in a 3:2 resonance.

Nature provides another clock suitable for planet hunting around another type of evolved object. Pulsations of certain white dwarf stars are highly stable $\left(\mathrm{d} P / \mathrm{d} t<10^{-14}\right)$ and their arrival times can be searched for planetary companions. Mullally et al. (2007) surveyed 15 white dwarfs and found evidence in one system for a $2 M_{\text {Jup }}$ companion in a $4.5 \mathrm{yr}$ orbit. If confirmed, this result will demonstrate that planets survive not just the red giant phase of stellar evolution (as demonstrated via radial velocity detections of a few planets around red giant stars), but also into the white dwarf phase. 


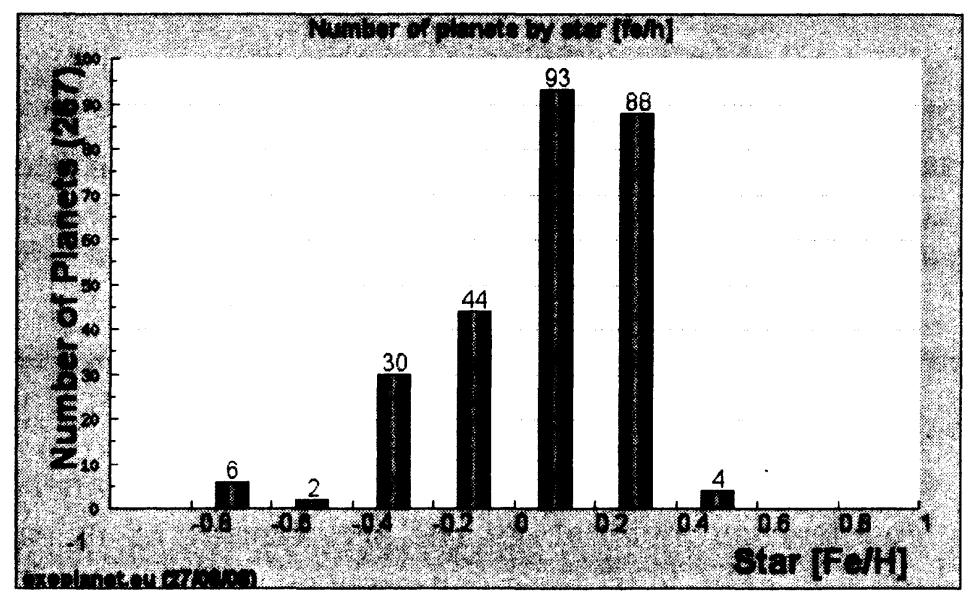

(a) Host Star Metallicity

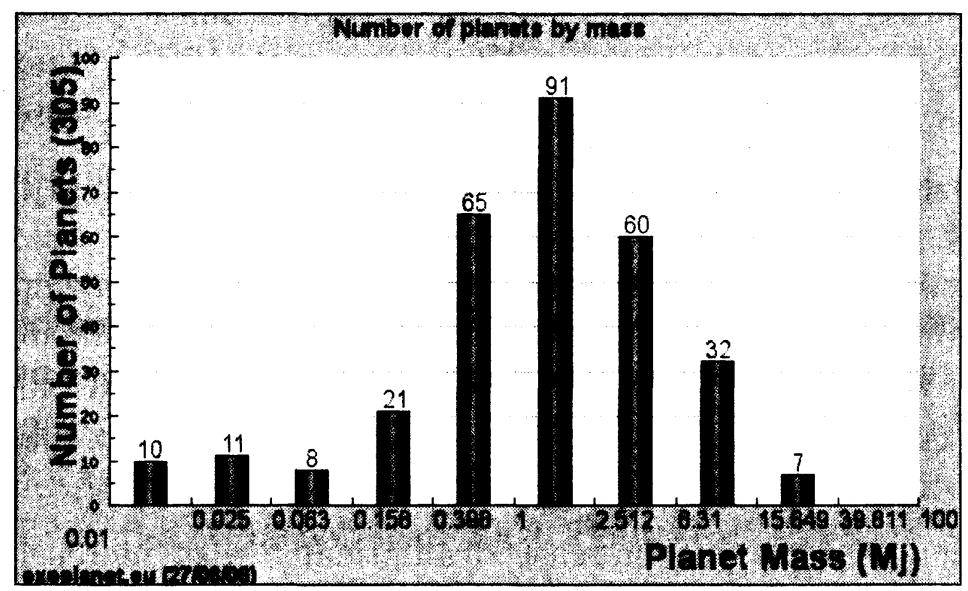

(b) Planet Mass

Fig. 2. The number of planets is sharply increasing function of host star metallicity (a) and of decreasing planet mass (b). Selection effects in the RV census account for the drop in the number of planets below $1 M_{J u p}$. Data and figure from the Exoplanet Encyclopedia (Schneider 2008).

\subsection{Radial Velocity and the Physics of Solar Systems}

The first detection of an exoplanet around a main sequence star came from the measurement of radial velocities (RV) of the star 51 Pegasi (Mayor \& Queloz 1995), a $0.47 M_{J u p}$ object in a surprising and not very habitable 4.2 day period. The RV technique has given us our most complete census of planetary systems, covering a period range from a few days to more than 10 years and a mass range starting at $<13 M_{\text {Jup }}$ of the largest gas giants, down through the icy giants and even into the super earth $\left(<10 M_{\oplus}\right)$ range. The basic relationship for $\mathrm{RV}$ amplitude, $K$, is given by (e.g. Cumming 2007):

$$
K\left(\mathrm{~m} \mathrm{~s}^{-1}\right)=\frac{28.4}{\sqrt{1-e^{2}}} M_{p, J u p} \sin (\mathrm{i}) P_{y r}^{-1 / 3} M_{*, \odot}^{-2 / 3}
$$


Table 1. Radial velocity signatures.

\begin{tabular}{|c|c|c|c|c|c|c|c|}
\hline \multirow[b]{2}{*}{ Star } & \multicolumn{3}{|c|}{ Stellar Properties (Solar Units) } & \multicolumn{2}{|c|}{ Planet Location } & \multirow{2}{*}{$\begin{array}{c}\text { Jupiter } \\
\text { Ampl. } \mathrm{m} \mathrm{s}^{-1}\end{array}$} & \multirow{2}{*}{$\begin{array}{c}\text { Earth } \\
\text { Ampl. } \mathrm{m} \mathrm{s}^{-1}\end{array}$} \\
\hline & Mass & Luminosity & Radius & $\begin{array}{c}\text { Dist } \\
(\mathrm{HZ}, \mathrm{AU})\end{array}$ & $\begin{array}{c}\text { Period } \\
(\mathrm{yr})\end{array}$ & & \\
\hline G2 & 1 & 1 & 1 & 1.00 & 1.00 & 28 & 0.09 \\
\hline K5 & 0.8 & 0.15 & 0.72 & 0.39 & 0.27 & 51 & 0.15 \\
\hline M5 & 0.27 & 0.011 & 0.21 & 0.10 & 0.07 & 169 & 0.51 \\
\hline L5 & 0.05 & 0.001 & 0.1 & 0.03 & 0.03 & 714 & 2.16 \\
\hline
\end{tabular}

for a planet of mass $M_{p}$ orbiting a star of mass $M_{*}$, with period $P$, eccentricity $e$ and inclination $i$. From the observations of more than 250 planets in more than 220 systems we have learned much about the architecture of at least the gas and icy giants (see Cumming et al. 2007 for a comprehensive summary and references):

- Approximately $10 \%$ of FGK stars have Jupiter mass planets with masses above $0.3 M_{J u p}$ and periods $<2000$ days with $10 \%$ or more in multiple systems.

- The incidence of planets increases sharply with decreasing planetary mass, roughly as $\mathrm{d} \log (N) / \mathrm{d} \log (M) \propto M^{-1.3 \pm 0.2}$.

- With the exception of objects in the closest, tidally-locked orbits which are expected to be circularized, the distribution of orbital eccentricities is very broad with circular orbits like those in our solar system the exception not the rule.

- The incidence of planets increase dramatically with increasing metallicity, $\propto[\mathrm{Fe} / \mathrm{H}]^{2}$ (Fischer \& Valenti 2005).

As described elsewhere in this conference, the frontier of RV measurements is advancing dramatically with observations breaking the $1 \mathrm{~m} \mathrm{~s}^{-1}$ barrier, measurement timelines now exceeding a decade, fainter stars and/or later spectral types now coming under scrutiny, and surveys with multi-object spectroscopy just beginning. These advances have led to record holders across planet parameter space: HD 154345 with a $2.2 M_{J u p}$ companion at 9.2 AU (Wright et al. 2007) and GL 581 with a $5.2 M_{\oplus}$ companion at $0.07 \mathrm{AU}$ (Udry et al. 2007). Only $3 \mathrm{M}$ stars have been found to host giant planets within 2.5 AU for a fractional incidence $\sim 2 \pm 1 \%$, much less than the incidence among higher mass stars (Butler et al. 2006; Johnson et al. 2007). Searches for lower mass planets are underway and in the long run searches with a precision of $0.5 \mathrm{~m} \mathrm{~s}^{-1}$ might someday find an earth in the habitable zone orbiting 0.1 AU from an M5 star (Table 2.2; Udry et al. 2007).

The ultimate limits to RV precision will probably not be instrumental as highly stable spectrographs like HARPS and possibly innovative laser-stabilized spectrographs ( $\mathrm{Li}$ et al. 2008) come into operation at the $<0.1 \mathrm{~m} \mathrm{~s}^{-1}$ level which is comparable to amplitude of an earth orbiting $1 \mathrm{AU}$ from a $\mathrm{G}$ star (Table 1). At this time, however, there is considerable controversy as to the ultimate limit of RV observations due to motions in the stellar photosphere. In the case of the most quiescent $\mathrm{K}$ and $\mathrm{M}$ stars it may be possible to push into the habitable zone for 
Table 2. Transit signatures.

\begin{tabular}{|c|c|c|c|c|c|}
\hline \multirow[b]{2}{*}{ Star } & \multicolumn{2}{|c|}{ Planet Location } & \multirow{2}{*}{$\begin{array}{c}\text { Duration } \\
\text { (hr) }\end{array}$} & \multirow{2}{*}{$\begin{array}{l}\text { Jupiter } \\
\text { Amplitude }\end{array}$} & \multirow{2}{*}{$\begin{array}{c}\text { Earth } \\
\text { Amplitude }\end{array}$} \\
\hline & Dist (HZ, AU) & Period (yr) & & & \\
\hline G2 & 1.00 & 1.00 & 13.00 & $1.0 \%$ & $0.01 \%$ \\
\hline K5 & 0.39 & 0.27 & 6.51 & $1.9 \%$ & $0.02 \%$ \\
\hline M5 & 0.10 & 0.07 & 1.70 & $22.7 \%$ & $0.23 \%$ \\
\hline L5 & 0.03 & 0.03 & 1.03 & $100.0 \%$ & $1.00 \%$ \\
\hline
\end{tabular}

earth-sized planets (Udry, this conference). For more typical stars the practical limits to RV may be much higher so that the detection of habitable earths orbiting nearby solar type stars may have to rely on other techniques, i.e. astrometry (Sect. 2.5). Nevertheless, RV observations continue to be the workhorse of the planet detection enterprise, expanding the census, as well as confirming and providing masses and orbits of transiting planets.

\subsection{Transits and the Physics of Planets}

The most dramatic results in recent exoplanet research have come from the measurements of transiting planetary systems, first as an adjunct to previously known radial velocity systems, e.g. the first transiting planet (HD 209458b; Henry et al. 2000; Charbonneau et al. 2000). Many transits are now coming directly from large-area ground-based surveys such as TrES, HAT, XO and WASP with many more discoveries expected from the space-based missions, CoRoT (CNES/ESA) and Kepler (NASA). See the proceedings of IAU 253 for a comprehensive review of recent transit results (Charbonneau 2008; Pont et al. 2008).

The transit effect is inherently simple (Perryman 2000):

$$
\begin{gathered}
\text { Depth }=\left(\frac{R_{p}}{R_{*}}\right)^{2} \\
\text { Duration }(\mathrm{hr})=\frac{\text { Period }}{\pi} \frac{R_{*} \cos \delta+R_{p}}{a} \sim 13 M_{*, \odot}^{-1 / 2} a_{A U}^{1 / 2} R_{*, \odot} \\
\text { Minimum Inclination } \sim \cos ^{-1} \frac{R_{*}}{a} \sim 88-90^{\circ} \\
\text { Probability of transit } \sim \cos \frac{R_{*}}{a} \sim 1-10 \%
\end{gathered}
$$

where $R_{p}$ and $R_{*}$ are the planeteray and stellar radii, $a$ the semi-major axis, and $\delta$ the transit impact parameter. But when this simple photometric effect is measured with extremely high precision, an abundance of information about the planet and the host star can be obtained (Seager \& Mallén-Ornelas 2003): the transit geometry removes the uncertainties in planetary properties due to unknown inclination; the combination of mass from RV and radius from the transit yields density, a key indicator of internal structure and thus of planetary formation and evolution scenarios (Fig. 3; Charbonneau 2008); precision photometry at primary transit 


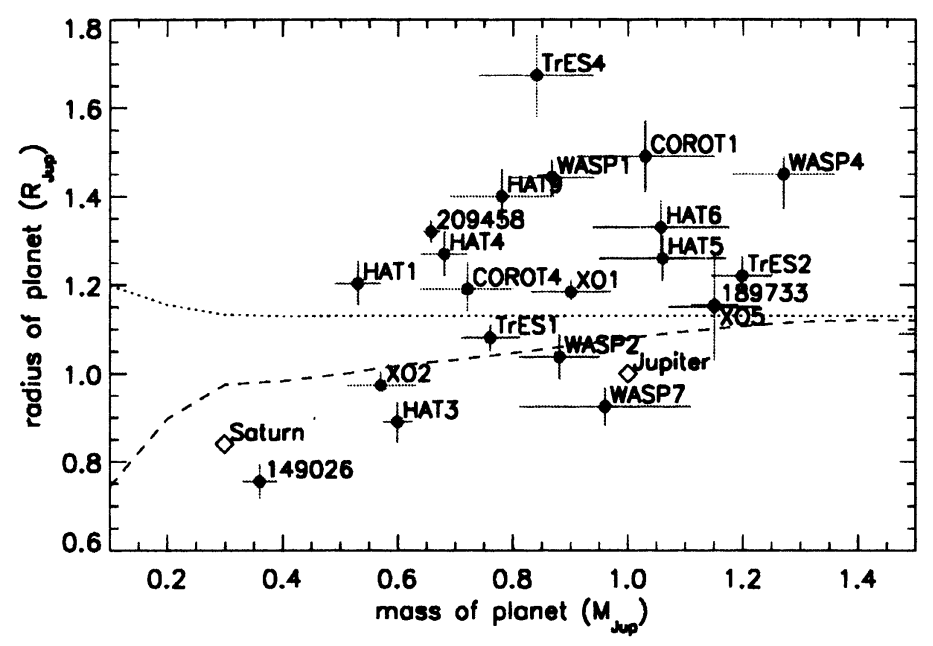

Fig. 3. The combination of transit and RV data yields the density of planets. The solid line indicates expected values for a $4.5 \mathrm{Gyr}$ planet with no heavy element core while the dashed line describes a model for an object with a $20 M_{\oplus}$ core (Bodenheimer et al. 2003). The wide range of values from the very dense planets (HD 19026) to the very puffy (TrES-4) is indicative of a variety of formation scenarios, e.g. different sized solid cores, and/or differing environmental conditions, e.g. effects of insolation or eccentricity damping (Charbonneau 2007; Fig. from Charbonneau 2008).

(planet goes in front of star) allows determination of atmospheric composition at visible wavelengths; precision photometry at secondary transit (planet goes behind star) allows infrared energy from the planet to be observed directly (Charbonneau et al. 2005; Deming et al. 2005), including emission in spectral lines (Richardson et al. 2008; Swain et al. 2008) which allows determination of atmospheric temperature structure and chemical composition (Fortney et al. 2008; Burrows et al. $2008)$; precision photometry throughout a planet's orbit yields a phase function (visible) or surface temperature maps (infrared) and constraints on global energy circulation (Fig. 4; Knutson et al. 2008); the timing of transits could reveal the presence of other planets through orbital interactions; and long term monitoring of transiting systems might reveal moons or rings around the known planet, or additional co-planar planets.

Some of the most remarkable results to date include: 1) the census of planetary density now being determined for over two dozen systems (Fig. 3) showing with many surprises, including very lightweight systems such as TrES-4 and remarkably dense planet orbiting HD 149026 with a massive $\left(70 M_{\oplus}\right)$ rocky core (Sato et al. 2005); the thermal mapping of HD 189733 with Spitzer (Knutson et al. 2008; Fig. 4a); and the detection of $\mathrm{CH}_{4}$ and $\mathrm{H}_{2} \mathrm{O}$ in $\mathrm{HD} 189733$ with HST (Fig. 4b; Swain et al. 2008). This is a remarkable yield of information in just one or two years with much more to come, particularly from space with the CoRoT (this conference) and Kepler, and from high precision measurements of the brightest systems using Spitzer, HST, and eventually the James Webb Space Telescope at resolutions of $\sim 500$ (Greene et al. 2007; Beichman et al. 2008). 


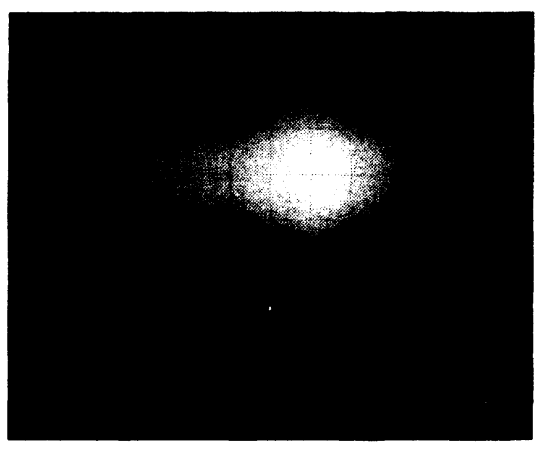

(a) Thermal map of HD $189733 \mathrm{~b}$

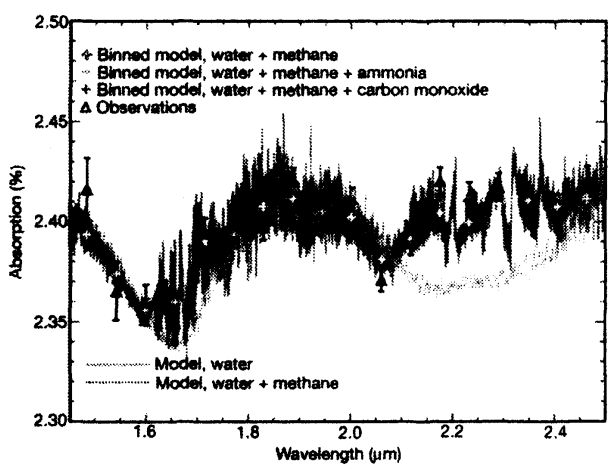

(b) Spectrum of Emission from HD 189733b

Fig. 4. a) Continuous monitoring of the infrared emission from HD $189733 \mathrm{~b}$ with the Spitzer Space Telescope has yielded a temperature map of the planet - a first for an object beyond the solar system. The temperature peak is displaced from the sub-stellar point indicating the presence of significant winds on the planet (Knutson et al. 2008). b) The spectrum of HD 189733 obtained with the Hubble Space Telescopes shows evidence for water and methane, the first detection of molecules in a planet beyond the solar system (Swain et al. 2008).

Finally, there is the exciting prospect of finding terrestrial-sized planets orbiting in the habitable zones of solar type stars (with Kepler) and finding similar planets orbiting low mass M stars with ground-based surveys, e.g. with the $M_{E a r t h}$ project (Irwin et al. 2008) or with eventual space based surveys such as TESS (Ricker 2008), PLATO or ASTrO (Beichman 2008). The transits of very late type stars are of such large amplitude (Table 2) that spectroscopic follow-up of super-Earths (few $R_{\oplus}$ ) with JWST is a realistic and exciting prospect. Large area (all-sky) surveys, first from the ground, e.g. Super-WASP, and ultimately from space (TESS, ASTrO), are very important since only such surveys can identify the closest and brightest transiting systems and thus provide targets amenable to high precision photometric and spectroscopic follow-up.

\subsection{Microlensing}

The gravitational bending of light produces remarkable effects on a wide range of distance and mass scales, but one scale is critical for exo-planet research. When a planet-bearing star serves as a lens to a more distant source star, the resultant amplification of the background star is modified from its simplest form by one or more features in the photometric time series. These features have an easily measurable amplitude of $\sim 1-10 \%$ that is independent of planet mass but whose duration and probability of detection scale as $\sqrt{M_{p}}$.

The scale of the Einstein lens, $R_{E}$, is given by

$$
R_{E}=\sqrt{\frac{4 G M}{c^{2}} D_{s} x(1-x)}=2.85 \mathrm{AU} \sqrt{M_{\oplus} D_{k p c}}
$$




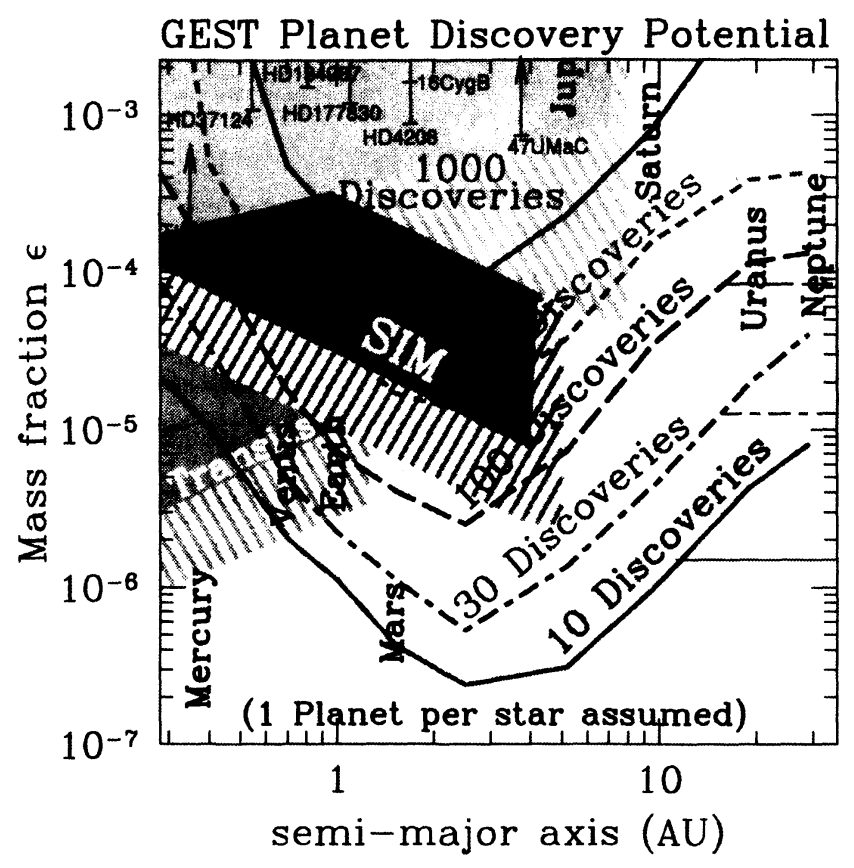

Fig. 5. A space mission (GEST) to monitor the Galactic Bulge region would find many thousands of planetary microlensing events, including many low mass planets (Bennett \& Rhie 2002).

where $x=D_{l} / D_{s}$ with $D_{l}$ and $D_{s}$ are the distances to the lens and source objects respectively and $D=\frac{D_{l}\left(D_{s}-D_{l}\right)}{D_{s}}$ (Mao \& Paczynski 1991; Gould \& Loeb 1992 and reviewed in Gould et al. 2007). The time for the lens star to traverse in front of the host, $t_{E} \sim R_{E} / v$, depends on the distances and relative velocities, but is of order 30 days at typical galactic velocities of $200 \mathrm{~km} \mathrm{~s}^{-1}$.

Networks of dozens of small telescopes are now in place to follow-up microlensing events with the high temporal cadence necessary to find planets. With six planetary events now well characterized, some trends are becoming clear: a wide range of planet masses can be detected, from $2.6 M_{J u p}$ to $0.017 M_{J u p}\left(=5.6 M_{\oplus}\right)$, spanning orbital distances from 2-5 AU (Gaudi et al. 2008). High magnification events from low impact parameter crossings increase the possibility of finding multiple planets and can be analyzed in sufficient detail to break many degeneracies in the theoretical analysis. One such system has been shown to consist of a $0.5 M_{\odot}$ star at $1.5 \mathrm{kpc}$ with 2 planets, 0.71 and $0.27 M_{J u p}$ in 2.3 and $4.6 \mathrm{AU}$ orbits (Gaudi et al. 2008). The fact that microlensing systems are finding as many low mass planets as high mass ones, despite the much higher detection probability for higher mass objects, suggests that earth mass objects in the 1-5 AU regime may be quite common (Gaudi et al. 2008).

A space mission such as GEST (Fig. 5; Bennett \& Rhie 2002) would find many thousands of microlensing systems, including many tens of terrestrial mass systems as well as free-floating planets, should such objects exist. The main disadvantage of all microlensing observations is that none can ever be followed up once the unique geometrical alignment ends after a month or so. 
Table 3. Astrometric signatures at $10 \mathrm{pc}$.

\begin{tabular}{lcccc}
\hline & \multicolumn{2}{c}{ Planet Location } & Jupiter & Earth \\
Star & Dist $(\mathrm{HZ}, \mathrm{AU})$ & Period $(\mathrm{yr})$ & Ampl $(\mu$ as $)$ & Ampl $(\mu$ as $)$ \\
\hline G2 & 1.00 & 1.00 & 96 & 0.29 \\
K5 & 0.39 & 0.27 & 47 & 0.14 \\
M5 & 0.10 & 0.07 & 37 & 0.11 \\
L5 & 0.03 & 0.03 & 61 & 0.18 \\
\hline
\end{tabular}

\subsection{The Astrometric Search for Planets}

While the magnitude of the astrometric signal is small (Table 3), the effect has very different selection biases from the radial velocity and transit measurements, making it a valuable complement to the other observations.

$$
\text { Amplitude }(\mu \mathrm{as})=500 \frac{M_{p, J u p}}{M_{*, \odot}} \frac{R_{A U}}{5.2 \mathrm{AU}} \frac{10 \mathrm{pc}}{\operatorname{Dist}(\mathrm{pc})} .
$$

Most importantly, as a two-dimensional position measurement, astrometry is independent of orbital inclination, so this ambiguity in the derivation of system properties is removed. Instead of the signal decreasing with orbital distance as is the case for RV, the astrometric signal increases in strength, although longer time baselines are required to measure a complete period. Unfortunately, the astrometric signal decreases with increasing distance to the star, so unlike RV, astrometric measurements of the smallest planets will be limited to the nearest stars, typically less than $100 \mathrm{pc}$.

Up until now the observational challenge from ground or space (with HST or Hipparcos) has been so great that there have as yet been no astrometric discoveries of planets, only false claims (Barnard's star; Gatewood \& Eichorn 1973), confirmations ( $\epsilon$ Eri; Benedict et al. 2006) and refutations of RV detections (HD 33636 for which astrometry showed that the RV-detected companion was not a planet, but rather an $M$ star in a near-face-on orbit; Bean et al. 2007). But the promise of astrometry is enormous since this technique may be pushed to extreme sensitivity levels from space and can unambiguously measure three crucial characteristics of planets: mass, the size and shape of its orbit (semi-major axis and eccentricity), and the inclination of its orbit which in the case of multiple planets tells if the orbits are co-planar. While large format CCD arrays and adaptive optics on large ground-based telescopes or long baseline interferometers (Keck-I or VLT-I) may enable astrometric measurements at the 30-100 $\mu$ as level (Pott et al. 2008; Geisler et al. 2006), the real promise of astrometry will not be realized until one can achieve the sub- $\mu$ as possible from space.

Early in the next decade, ESA's GAIA mission will carry out an astrometric sky survey with a magnitude-dependent mission accuracy of $\sim 10 \mu$ as between $8<V<$ $15 \mathrm{mag}$, degrading to brighter and fainter magnitudes. GAIA will detect hundreds of gas giants around stars out to $100 \mathrm{pc}$. This first astrometric reconnaissance will include many stars not suitable for RV observations, such as young stars and white dwarfs (Table 4). But the second step in astrometry must be a mission capable of much higher precision and cadence. Presently under discussion is the 
Table 4. Planet surveys with SIM and GAIA.

\begin{tabular}{|c|c|c|c|}
\hline & SIM Deep Survey & SIM Broad Survey & GAIA \\
\hline $\begin{array}{l}\text { Single Measurement } \\
\text { Accuracy }\end{array}$ & $1 \mu$ as & $4 \mu \mathrm{as}$ & $70 \mu$ as \\
\hline $\begin{array}{l}\text { Mission Measurement } \\
\text { Accuracy }\end{array}$ & $<0.1 \mu$ as $(2502 \mathrm{D}$ obs $)$ & $<0.4 \mu$ as $(1002 \mathrm{D}$ obs $)$ & $10 \mu$ as $(75$ obs $)$ \\
\hline Target V Range (mag) & -1 to 7 & -1 to 15 & $\begin{array}{c}7 \text { to } 15 \mathrm{mag} \\
V<7 \mathrm{mag} \text { at } \\
\text { reduced sensitivity }\end{array}$ \\
\hline Science Goals & $\begin{array}{c}\text { Earth Analog Survey } \\
\text { in } \mathrm{HZ} \text { of } 50 \sim 190 \text { closest } \\
\text { bright stars }\left(1-3 M_{\oplus}\right)\end{array}$ & $\begin{array}{l}\text { Survey } 1000 \text { stars } \\
\text { for icy/gas giants } \\
\text { Uranus-Jupiter }\end{array}$ & $\begin{array}{l}\text { All sky survey } \\
\text { for gas giants } \\
\text { Saturn/Jupiter }\end{array}$ \\
\hline
\end{tabular}

Table 5. Planet mass-limited surveys with SIM-lite.

\begin{tabular}{|c|c|c|c|}
\hline & \multirow{2}{*}{$\begin{array}{l}\text { Limiting Mass } \\
\text { Sensitivity }\end{array}$} & \multicolumn{2}{|c|}{ \# of stars } \\
\hline & & $40 \%$ of Mission time & $80 \%$ of Mission time \\
\hline Survey 1 & $1.0 M_{\oplus}$ & 49 & 67 \\
\hline Survey 2 & $2.0 M_{\oplus}$ & 114 & 155 \\
\hline Survey 3 & $3.0 M_{\oplus}$ & 187 & 255 \\
\hline
\end{tabular}

SIM-Lite utilizes $50 \mathrm{~cm}$ optics on a $6 \mathrm{~m}$ baseline.

Space Interferometer Mission (SIM; Unwin et al. 2008) which will provide a limiting astrometric accuracy over a narrow field $<0.1 \mu$ as making SIM capable of detecting terrestrial-mass planets in the habitable zones of tens to hundreds of stars. While the final instrumental properties of SIM and survey strategy are still being debated, Table 5 and Figure 6 show the promise of "SIM-Lite", a version of SIM with a $6 \mathrm{~m}$ baseline and $50 \mathrm{~cm}$ optics.

SIM's narrow-angle astrometry of each target star will be made relative to at least 3 reference stars selected to surround the target star within $\sim 1^{\circ}$. A single measurement will achieve $0.85 \mu$ as differential measurement precision for $V=7 \mathrm{mag}$ stars, including instrumental and photon-limited errors. Recent laboratory measurements indicate that SIM's systematic noise floor is below $0.1 \mu$ as after many repeated measurements. Monte Carlo simulations show that the minimum planetary amplitude that can be detected reliably ( $<1 \%$ False Alarm Probability) is given by $5.8 \frac{\text { Single Measurement Accuracy }}{\sqrt{N_{\text {epoch }}}}$. For $N_{\text {epoch }}=250$ SIM's reliable detection limit is $\sim 0.1 \mu$ as, well below the $0.3 \mu$ as signature of an Earth analog orbiting a solar type star at 10 pc. A detailed analysis of a list of over 300 nearby stars shows that SIM-Lite could survey between 50-187 of the closest stars (depending on desired limiting mass sensitivity) in $40 \%$ of the mission time (Table 5; Tanner et al. 2006). Starspots and other manifestations of stellar variability can affect the astrometric centroid resulting in a jitter similar to the spectroscopic noise that limits radial velocity precision. Fortunately, these effects in white light are reduced so that they will not be a primary limitation for the detection of terrestrial planets around solar type stars (Unwin et al. 2008; Catanzarite et al. 2006).

SIM will also be able to survey a larger number (1000-2000) stars at lower precision ( $\sim 4 \mu \mathrm{as}$, single measurement accuracy), adequate to survey a wide variety of stellar types not amenable to radial velocity observation, such as $\mathrm{O}$, B and A spectral types, white dwarfs, low metallicity stars, and young stars 


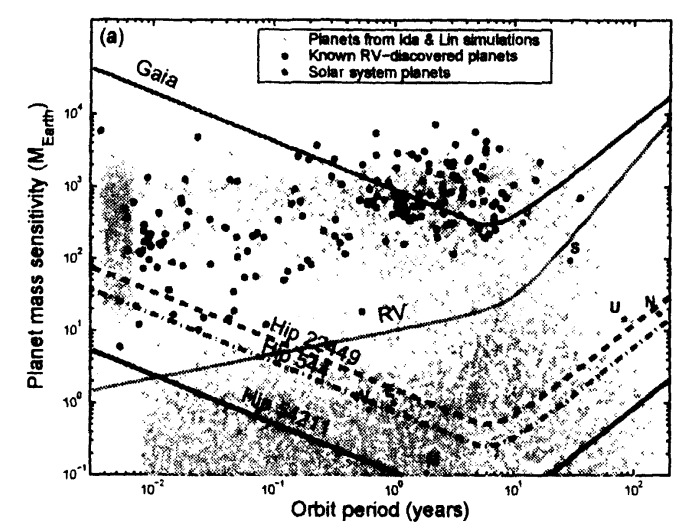

(a) Deep Survey for Planets

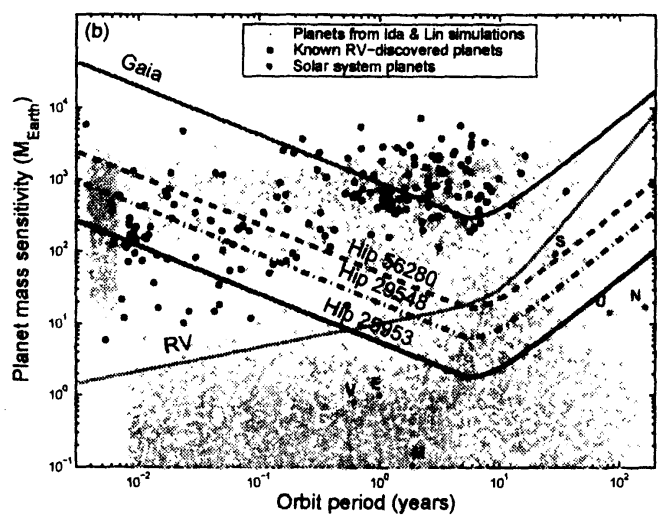

(b) Shallow Survey for Planets

Fig. 6. a) The discovery space for rocky Earth-like $\left(\sim 1-10 M_{\oplus}\right)$ planets in the habitable zone ( 0.7-1.5 AU for a G star); b) a comparable plot for the "Broad Survey" obtained with $4 \mu$ as accuracy. In both cases, the small dots represent a theoretical planet distribution (Ida \& Lin 2005; see Sect. 3) for planets of 0.1-3000 $M_{\oplus}$. Exoplanets discovered before early 2007 and with semi-major axes $>0.03 \mathrm{AU}$ are shown as filled circles. Labeled curves represent the estimated sensitivity limits of indirect detection methods: for radial velocity method ( $\mathrm{RV}$ at $1 \mathrm{~m} \mathrm{~s}^{-1}$ ), and astrometry with SIM and Gaia. The SIM sensitivity in this space is a broad band, defined by the three lowest curves (labeled with specific Hipparcos star numbers). The lowest curve shows the "best" star (as computed from star mass and distance); the middle curve represents the median star; and the upper curve shows the least favorable star in the sample. Also shown is the effective sensitivity of Gaia for stars at $50 \mathrm{pc}$, a typical distance for Gaia targets.

(Beichman 2001; Tanner et al. 2007). Even at its lower level of precision, SIM's shallow survey will be approximately 25 times deeper than GAIA's all-sky survey, meaning that SIM will reach down to the level of icy giants whereas GAIA is likely to reach only to gas giants for most of its stars.

\section{Theory Rushes to Keep Up}

From the initial discovery of pulsar planets (extremely unexpected), the first exoplanets in $\sim 4$ day orbits (very unexpected), and the wide range of eccentricities 
(just plain unexpected) theoreticians have been rushing to keep up with the observations. A variety of formation mechanisms, including accretion and runaway growth in a disk (Ida \& Lin 2005) on the one hand or multiple fragmentation events in a gaseous disk on the other (Boss et al. 2001) have been proposed and compared with the ever expanding body of observations. The strong dependence of the incidence of planets on stellar metallicity seems to support the accretion models, but much remains to be done to arrive at a consistent model based on full physical understanding of the physical sources of viscosity and resultant migration in the disk (Ida \& Lin 2008).

Figure 6 suggests 5 main groupings of planets (Ida \& Lin 2005): gas giants $\left(100-1000 M_{\oplus}\right)$ formed beyond and still resident outside the ice-line; icy giants $\left(10-100 M_{\oplus}\right)$ located well beyond the ice-line whose growth is truncated by the long time scales, low surface density, and rapid dissipation of the gaseous disk; rocky planets $\left(0.1-10 M_{\oplus}\right)$ throughout the inner solar system; "warm" gas and icy giants spread throughout the inner solar system by either migration or orbital interactions; and "hot" gas or icy giants piled up in tidally-circularized $\sim 4$ day orbits, whose migration was apparently halted in the nick of time by the termination of disk at the dust sublimation radius.

But many questions remain and the existing theories may be the result of trying to explain selection effects of the RV and transit observations which both favor large planets in close orbits: is the gap between 10 and $100 M_{\oplus}$ real? Is the pile-up of hot planets as dramatic is suggested in the figure? What is the incidence of terrestrial and lower mass rocky planets? What accounts for the wide range of eccentricities? How does the incidence of planets of various size depend on the masses of the parent star and protostellar disk? And most importantly for the search for habitable planets and life: what is the incidence of potentially habitable earth-sized planets $\left(0.5-5 M_{\oplus}\right)$ located in or near the habitable zone $\left(\sim 1 \sqrt{L_{\odot}} \mathrm{AU}\right)$ ?

These questions about the architecture of planetary systems demand an improved census of planets from transit missions for the innermost regions (0.05$1 \mathrm{AU}), \mathrm{RV}$ and astrometry for low mass sensitivity in the mid-to-outer regions (0.5-10 AU), and direct imaging for the outer reaches of planetary systems (at least for young systems where planets might be detectable). Complete orbital information from astrometry, including information on coplanarity in multiple systems, will be particularly important in understanding formation mechanisms and the effects or orbital interactions.

\subsection{Direct Detection}

Direct detection of a spatially resolved solar system is a compelling long-term goal for exoplanet research, for both the intense public interest that would accompany such an image and the scientific prospect of obtaining spectroscopic information on individual planets. In particular, direct spectroscopy is necessary for planets too far from their host stars to be detected via the transit technique. But obtaining such images will be technologically extremely challenging. The star-planet flux ratios are extreme, $10^{10}$ (visible) and $10^{7}$ (infrared), for an earth at $1 \mathrm{AU}$ from a 


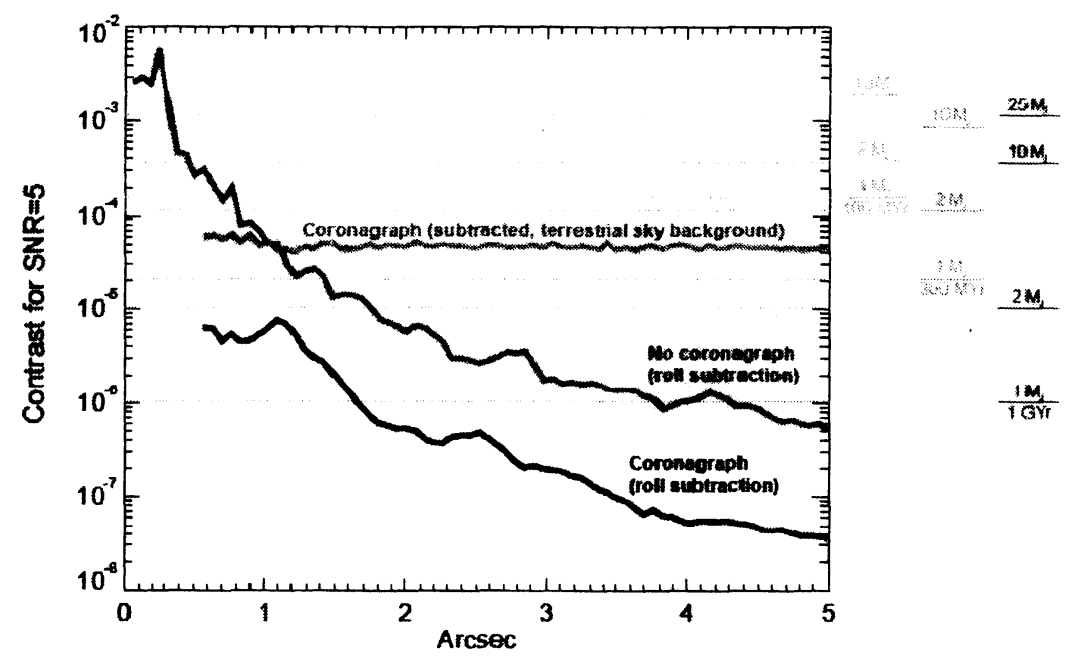

Fig. 7. The sensitivity of NIRCAM on JWST at $4.5 \mu \mathrm{m}$ is shown with successive degrees of coronagraphic suppression and is compared with a telescope limited by terrestrial backgrounds. On the right hand side of the figure, the contrast level of planets of various masses and ages is shown relative to an M0 star at 4 pc (Krist et al. 2007).

G2 star; the signal levels are low, a few photons $\mathrm{m}^{-2} \mathrm{~s}^{-1}$ at $10 \mathrm{pc}$; and the angular separations small, 10-100 mas to resolve $1 \mathrm{AU}$ at $10 \mathrm{pc}$. Progress in imaging will be incremental with gas giants studied first, initially from the ground, followed by space missions capable of studying terrestrial planets. The present and future state of the art in coronagraphy is discussed in a recent conference Proceedings of the conference In the Spirit of Bernard Lyot: The Direct Detection of Planets and Circumstellar Disks in the 21st Century (Kalas 2007).

\subsubsection{Gas Giants}

Young Jupiters with ages $<1$ Gyr have significant reservoirs of internal gravitational energy and correspondingly emit hundreds to thousands of times more infrared energy than mature planets heated only by faint starlight (Saumon et al. 1996). The atmospheric opacity of these planets results in complex spectral energy distributions with some wavelengths having much more emission than might be accounted for by a blackbody at an single effective temperature. Such features are particularly prominent at $5 \mu \mathrm{m}$.

Coronagraphs on large ground-based telescopes (5-10 m) are making the first discoveries of faint companions to nearby young stars (2MASS $1207 \mathrm{~b}$; Chauvin et al. 2004) with contrast ratios in the $10^{-4}-10^{-5}$ range for objects located tens of AU from their parent stars. This instrumental capability suffices to find objects of a 1-10 $M_{J u p}$ range, depending on the age of the planet. A few objects have been found and confirmed to be companions to a brighter star via their common proper motion. What remains controversial is their identification as planets $\left(<13 M_{J u p}\right.$; the deuterium burning limit), as opposed to brown dwarfs $\left(13<M<70 M_{J u p}\right)$ or even low mass stars $\left(>70 M_{\text {Jup }}\right)$. The relations between near-IR brightness, age, and mass are quite uncertain and dynamical mass determinations are impractical 


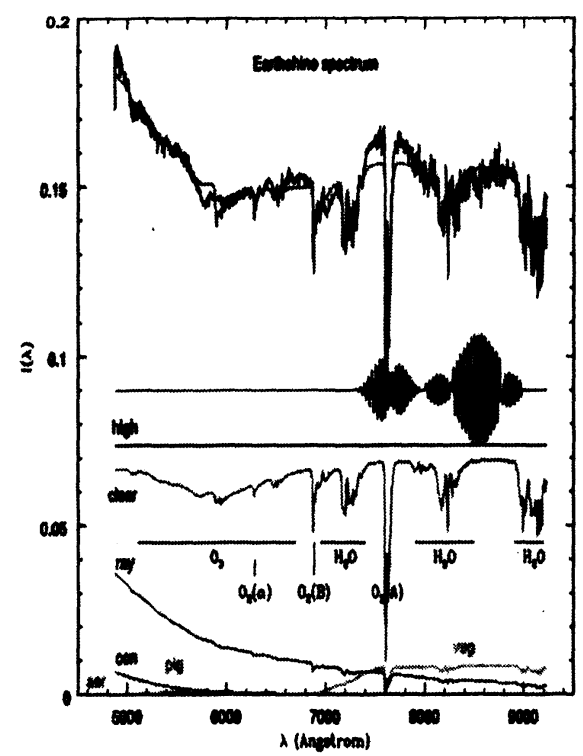

(a) Visible Spectrum of Earth

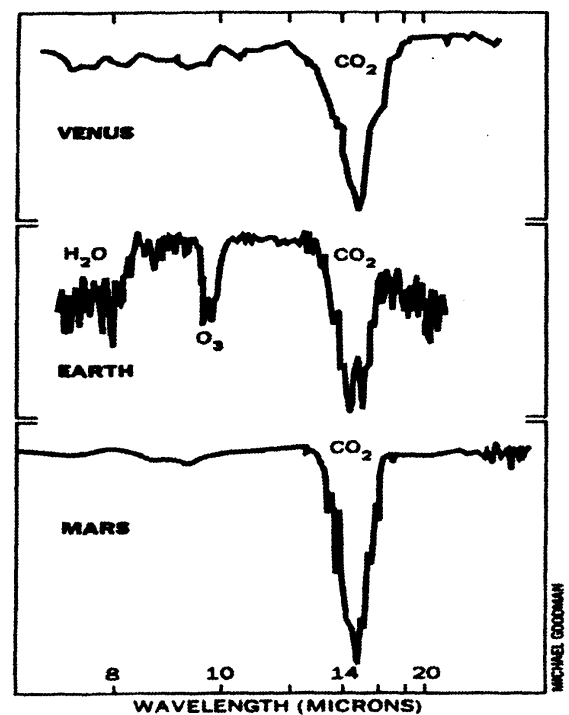

(b) Infrared Spectra Terrestrial Planets

Fig. 8. a) A visible spectrum of the Earth measured in earthshine from the moon shows numerous spectral features, including $\mathrm{O}_{2}$ and $\mathrm{H}_{2} \mathrm{O}$ that might someday be observed in distant exoplanets (Woolf et al. 2002); b) the infrared spectra of the terrestrial planets in our solar system show $\mathrm{CO}_{2}$ in all cases, but $\mathrm{H}_{2} \mathrm{O}$ and $\mathrm{O}_{3}$ are seen only in the Earth's atmosphere.

for objects on long period orbits. The next generation of coronagraphs, e.g. the Gemini Planet Imager (Macintosh et al. 2007), will push this contrast ratio to $10^{-6}-10^{-7}$ range with modest improvements in inner working angle. With advanced instruments operating on extremely large, diffraction limited telescopes (30-40 $\mathrm{m})$, it should be possible to image young, gas-giant planets orbiting within $2-3 \mathrm{AU}$ of the closest young stars $(25-50 \mathrm{pc})$, and possibly even detect mature planets orbiting the nearest, low mass stars $(<5 \mathrm{pc})$ where the contrast ratio is favorable, e.g. GL 876 and GL 3522.

An important adjunct of this program will be provided by the James Webb Space Telescope (JWST) whose near-infrared coronagraph will not offer great angular resolution or high contrast, but will provide great sensitivity at $3-5 \mu \mathrm{m}$. JWST will be able to detect 1 Gyr Jupiters in few AU orbits around nearby M stars and gas/icy giants orbiting at tens of $\mathrm{AU}$ around the nearest young stars (Krist et al. 2007). A highly optimized $2 \mathrm{~m}$ space telescope with an advanced coronagraph could find a few dozen mature planets in reflected light and carry out a limited program of spectroscopy (Trauger et al. 2007; Beichman et al. 2008).

\subsubsection{Terrestrial Planets and the Search for Life}

The scientific and technological community have expended much energy in the past decade considering the requirements for missions to detect directly emission 
Table 6. Measurement synergy for TPF-C, TPF-I/Darwin and SIM.

\begin{tabular}{|c|c|c|c|}
\hline & SIM & TPF-C & TPF-I \\
\hline \multicolumn{4}{|l|}{ Orbital Parameters } \\
\hline Stable orbit in habitable zone & Measurement & Measurement & Measurement \\
\hline \multicolumn{4}{|l|}{ Characteristics for Habitability } \\
\hline Planet temperature & Estimate & Estimate & Measurement \\
\hline $\begin{array}{l}\text { Temperature variability due to } \\
\text { eccentricity }\end{array}$ & Measurement & Measurement & Measurement \\
\hline Planet radius & Cooperative & Cooperative & Measurement \\
\hline Planet albedo & Cooperative & Cooperative & Cooperative \\
\hline Planet mass & Measurement & Estimate & Estimate \\
\hline Surface gravity & Cooperative & Cooperative & Cooperative \\
\hline Atmospheric and surface composition & Cooperative & Measurement & Measurement \\
\hline Time-variability of composition & & Measurement & Measurement \\
\hline Presence of water & & Measurement & Measurement \\
\hline \multicolumn{4}{|l|}{ Solar System Characteristics } \\
\hline $\begin{array}{l}\text { Influence of other planets, orbit } \\
\text { coplanarity }\end{array}$ & Measurement & Estimate & Estimate \\
\hline $\begin{array}{l}\text { Comets, asteroids, } \\
\text { and zodiacal dust }\end{array}$ & & Measurement & Measurement \\
\hline \multicolumn{4}{|l|}{ Indicators of Life } \\
\hline Atmospheric biomarkers & & Measurement & Measurement \\
\hline $\begin{array}{l}\text { Surface biosignatures (red edge of) } \\
\text { vegetation }\end{array}$ & & Measurement & \\
\hline
\end{tabular}

"Measurement" indicates a directly measured quantity from a mission.

"Estimate" indicates that a quantity that can be estimated from a single mission.

"Cooperative" indicates a quantity that is best determined from several missions.

from terrestrial planets with a particular goal of achieving spectroscopic resolutions adequate to assess the presence of atmospheric signposts of habitable environments and even of life itself. There is not sufficient space to summarize all this work, except to note physics experiments in star light nulling at the appropriate contrast level and inner working angle have been demonstrated in the laboratory (Trauger \& Traub 2007; Lawson et al. 2006) and that engineering solutions exist to all the challenges. A visible light telescope in the $4 \mathrm{~m}$ class operating with an internal coronagraph (Terrestrial Planet finder, TPF-C; Traub et al. 2006, 2008) or an external occulter (TPF-O; Cash et al. 2006) would search the habitable zones of $\sim 25$ stars to the terrestrial planet level with spectroscopy of species such as $\mathrm{H}_{2} \mathrm{O}, \mathrm{CO}_{2}$, and $\mathrm{O}_{2}$ possible for the brightest objects (Fig. 8a). An $8 \mathrm{~m}$ telescope would be even more powerful, allowing searches for and detailed characterization of $50 \sim 100$ stars.

A complementary capability to observe nearby stars with an infrared interferometer using 2-4 m telescopes on separated spacecraft with a baseline of $100 \mathrm{~m}$, i.e. NASA's TPF-I and ESA's Darwin projects, would produce data on planet's effective temperature, albedo, and identify spectral features such as $\mathrm{H}_{2} \mathrm{O}, \mathrm{CO}_{2}$, $\mathrm{O}_{3}$ and possibly $\mathrm{CH}_{4}$ (Fig. 8b; Beichman et al. 2006; Fridlund et al. 2006). An overview of relevant biomarkers in the visible and IR wavebands can be found in a number of papers and references therein (Des Marais et al. 2002; Kaltenegger \& Selsis 2007; Kaltenegger et al. 2006).

Ultimately, the combination of dynamical information from SIM's astrometry, visible light spectroscopy with TPF-C or TPF-O, and infrared information 
from TPF-I/Darwin will provide the complete characterization of nearby planets (Table 6; Beichman et al. 2006) and allow the first search for life beyond the solar system.

\section{Conclusion}

The growth of exoplanet research has been astounding: from no knowledge of planets beyond the solar system barely 15 years ago, to today's wealth of data, to tomorrow's dramatic promises. The progression from larger planets to smaller, from "hot" temperatures to "habitable zones", from single color photometry to spectroscopy has been and will continue to be steady. Ground-based observations will be important for transit surveys and radial velocity measurements as well as for eventual direct imaging of giant planets. Near-term space missions such as CoRoT, Kepler and JWST will play a vital role in expanding our knowledge about a wide variety of individual planets and planetary systems. Data from a combination of missions like SIM, TPF-C/O and TPF-I/Darwin will be needed to complete the characterization of our nearest neighbors and to search for life. These missions will not come quickly since the technological challenges are daunting and the individual missions expensive. But at the conclusion of such a program, we would be well along the way to addressing one of mankind's deepest and most long-lasting questions: "Are We Alone?"

\section{References}

Aubin, D., Workshop at the Denklabor Villa Garbald, Castasegna, Switzerland 2007, www. histnet.cnrs.fr/research/nadirane/IMG/doc/ALL_ABSTRACTS.bis.doc

Bean, J.L., McArthur, B.E., Benedict, G.F., et al., 2007, AJ, 134, 749

Beichman, C.A., 2001, Young Stars Near Earth: Progress and Prospects, ASP 244. ed. R. Jayawardhana and T. Greene, Conference Series San Francisco: Astron. Soc. Pac. Conf., 376

Beichman, C.A., Fridlund, M., Traub, W.A., et al., 2006, in Protostars and Planets V, ed. B. Reipurth, D. Jewitt, and K. Keil (University of Arizona Press, Tucson)

Beichman, C., 2008, in Transiting Planets, ed. F. Pont et al., Proceedings of IAU, 253 (Cambridge Univ. Press), in press

Benedict, F., et al., AJ, 132, 2206

Bennett, D., \& Rhie, S.H., 2002, ApJ, 574, 985

Bodenheimer, P., Laughlin, G., \& Lin, D.N.C., 2003, ApJ, 592, 555

Boss, A.P., 2001, ApJ, 551, L167

Bruno, G., 1584, De L'infinito Universo E Mondi

Burrows, A., Budaj, J., \& Hubeny, I., 2008, ApJ, 678, 1436

Butler, R.P., Johnson, J.A., Marcy, G.W., et al., 2006, PASP, 118, 1685

Cash, W., Schindhelm, E., Arenberg, J., et al., 2006, in Space Telescopes and Instrumentation I: Optical, Infrared, and Millimeter, ed. J.C. Mather, H.A. MacEwen, M.W.M. de Graauw, Proc. SPIE, 6265, 62651V 
Catanzarite, J., Law, N., \& Shao, M., 2008, Astronomical Instrumentation, Proceedings of June 2008 SPIE (Marseille France) [eprint arXiv:0807.1930], in press

Charbonneau, D., Brown, T.M., Latham, D., \& Mayor, M., 2000, ApJ, 529, L45

Charbonneau, D., et al., 2005, ApJ, 626, 523

Charbonneau, D., et al., 2007, Protostars and Planets V, 701

Charbonneau, D., 2008, in Transiting Planets, ed, F. Pont et al., Proceedings of IAU, 253 (Cambridge Univ. Press), in press

Chauvin, G., Lagrange, A.-M., Dumas, C., et al., 2004, A\&A, 425, L29

Cumming, A., Butler, R.P., Marcy, G.W., et al., PASP, 120, 867, 531

Des Marais, D., et al., 2002, Astrobiology, 2, 153

Deming, D., et al., 2005, Nature, 434, 740

Drake, F., \& Sobel, D., Is Anyone Out There? the Scientific Search for Extraterrestrial Intelligence, (Delacorte Press, New York, New York)

Fischer, D., \& Valenti, J., 2005, ApJ, 622, 1102

Fortney, J.J., Lodders, K., Marley, M.S., \& Freedman, R.S., ApJ, 678, 1419

Fridlund, C.V.M., d'Arcio, L., den Hartog, R., \& Karlsson, A., 2006, in Advances in Stellar Interferometry, ed. Monnier, J.D. Schöller, M. Danchi, W.C. Proc. SPIE, $6268,62680 \mathrm{R}$

Gatewood, G., \& Eichorn, H., 1973, AJ, 78,769

Gaudi, S., et al., 2008, PLANET and RoboNet Consortia, Science, 319, 927

Geisler, R., Setiawan, J., \& Henning, Th., 2008, Proceedings of the International Astronomical Union, IAU Symp., 249, 61

Gould, A., \& Loeb, A., 1992, ApJ, 396, 104

Gould, A., Gaudi, B.S., \& Bennett, D., 2007, White Paper submitted to NASA Exoplanet Forum [e-print arXiv:0704.0767]

Greene, T., Beichman, C.A., Eisenstein, D., et al., 2007, Techniques and Instrumentation for Detection of Exoplanets III, ed. D.R. Coulter, Proc. SPIE: 6693, 66930G

Henry, G., Marcy, G., Butler, R.P., \& Vogt, S.S., 1999, IAU Circ. 7307

Huygens, C., 1698, Cosmotheoros: or Conjectures concerning the Planetary Worlds, (London)

Ida, S., \& Lin, D.N.C., 2005, ApJ, 626, 1045

Ida, S., \& Lin, D.N.C., 2008, ApJ, 673, 487

Irwin, J., Charbonneau, D., Nutzman, P., \& Falco, E., 2008, in Transiting Planets ed. F. Pont et al., Proc. of IAU, 253 (Cambridge Univ. Press), in press [eprint arXiv:0807.1316]

Johnson, J.A., et al., 2007, ApJ, 670, 833

Kalas, editor, Proceedings of the conference In the Spirit of Bernard Lyot, The Direct Detection of Planets and Circumstellar Disks in the 21st Century, University of California, Berkeley, CA, USA

Kaltenegger, L., \& Selsis, F., 2007, in Extrasolar Planets. Formation, Detection and Dynamics, ed. R. Dvorak, 79

Kaltenegger, L., Jucks, K., \& Traub, W., 2006, in Direct Imaging of Exoplanets, Science \& Techniques, ed. C. Aime, and F. Vakili, Proc. IAU Colloquium 200 (Cambridge, UK: Cambridge University Press), 259 
Knutson, H., Charbonneau, D., Cowan, N.B., et al., 2008, ApJ, in press [arXiv:0802.1705]

Konacki, M., \& Wolszczan, A., 2003, ApJ, 591, L147

Krist, J.E., Beichman, C.A., Trauger, J.T., et al., in Techniques and Instrumentation for Detection of Exoplanets III. ed. Coulter, R. Daniel, Proc. SPIE, 6693, 66930H$66930 \mathrm{H}-12$

Lawson, P.R., Ahmed, A., Gappinger, R.O., et al., 2006, in Advances in Stellar Interferometry, ed. J.D. Monnier, M. Schöller, W.C. Danchi, Proc. SPIE, 6268, 626828

Li, Chih-Hao, et al., 2008, Nature, 452, 610

Macintosh, B., \& the GPI Consortium 2007, in Proceedings of the conference In the Spirit of Bernard Lyot, The Direct Detection of Planets and Circumstellar Disks in the 21st Century, ed. Paul Kalas (University of California, Berkeley, CA, USA)

Mao, S., \& Paczynski, B., 1991, ApJ, 374, L37

Mayor, M. \& Queloz, D., 1995, Nature, 378, 355

McKee, C., \& Taylor, J., 2001, Astronomy and Astrophysics in the New Millennium (National Academy Press, Washington, DC), 53

Mullally, F.R., 2007, Ph.D. Thesis, Univ. of Texas

Nordman, C., 1902, Comptes Rendus Acad.Sci., 134, 273, Reprinted in English in "Classics in Radio Astronomy" by W.T. Sullivan (Reidel, 1982), see [http://www.nrao.edu/whatisra/hist_prehist.shtml]

Perryman, M.A.C., 2000, Reports Progr., Phys., 63, 1209

Proceedings of IAU 253: Transiting Planets, 2008, ed. F. Pont, et al. (Cambridge Univ. Press), in press

Pott, J.-U., Woillez, J., Wizinowich, P., et al., 2008, Bull AAS, AAS Meeting 211, 160.17

Richalet, J-P., 2001, High Alt. Med. Biol., 2, 57

Richardson, L.J., Harrington, J., Seager, S., \& Deming, D., 2008, ApJ, 649, 1043

Ricker, G., et al., 2008, in Transiting Planets, Proceedings of IAU 253, ed. F. Pont et al. (Cambridge Univ. Press), in press

Sato, et al., for the N2K Consortium 2005, ApJ, 633, 465

Saumon, D., Hubbard, W.B., Burrows, A., et al., 1996, ApJ, 460, 993

Schneider, J., 2008 [http://www.exoplanet.eu]

Seager, S., \& Mallén-Ornelas, G., 2003, ApJ, 585, 1038

Swain, M.R., Vasisht, G., \& Tinetti, G., 2008., Nature, 452, 329

Swain, M.R., Bouwman, J., Akeson, R.L., et al., 2008, ApJ, 674, 482

Tanner, A.M., Catanzarite, J., \& Shao, M., 2006, in Advances in Stellar Interferometry, ed. J.D. Monnier, M. Schöller, W.C. Danchi, Proc. SPIE, 6268, 626847

Tanner, A.M., et al., 2007, PASP, 119, 747

Tarter, J., et al., 2007, Astrobiology, 7, 30

Traub, W.A., et al., 2006, in Advances in Stellar Interferometry, ed. J.D. Monnier, M. Schöller, W.C. Danchi, Proc. SPIE, 6268, 62680T

Traub, W.A., Shaklan, S. \& Lawson, P., in Proceedings of the conference In the Spirit of Bernard Lyot, The Direct Detection of Planets and Circumstellar Disks in the 21st Century, ed. P. Kalas (University of California, Berkeley, CA, USA) 
Trauger, J.T., et al., 2007, in Proceedings of the conference In the Spirit of Bernard Lyot, The Direct Detection of Planets and Circumstellar Disks in the 21st Century, ed. P. Kalas (University of California, Berkeley, CA, USA)

Trauger, J.T., \& Traub, W.A., 2007, Nature, 446, 771

Udry, S., et al., 2007, A\&A, 469, 43

Unwin, S., et al., 2007, PASP, 120, 38

Wolsczan, A., \& Frail, D., 1992, Nature, 355, 145

Woolf, N.J., Smith, P.S., Traub, W.A., et al., 2002, ApJ, 574, 430

Wright, J.T., Marcy, G.W., Fischer, D.A. et al., 2007, ApJ, 657, 533

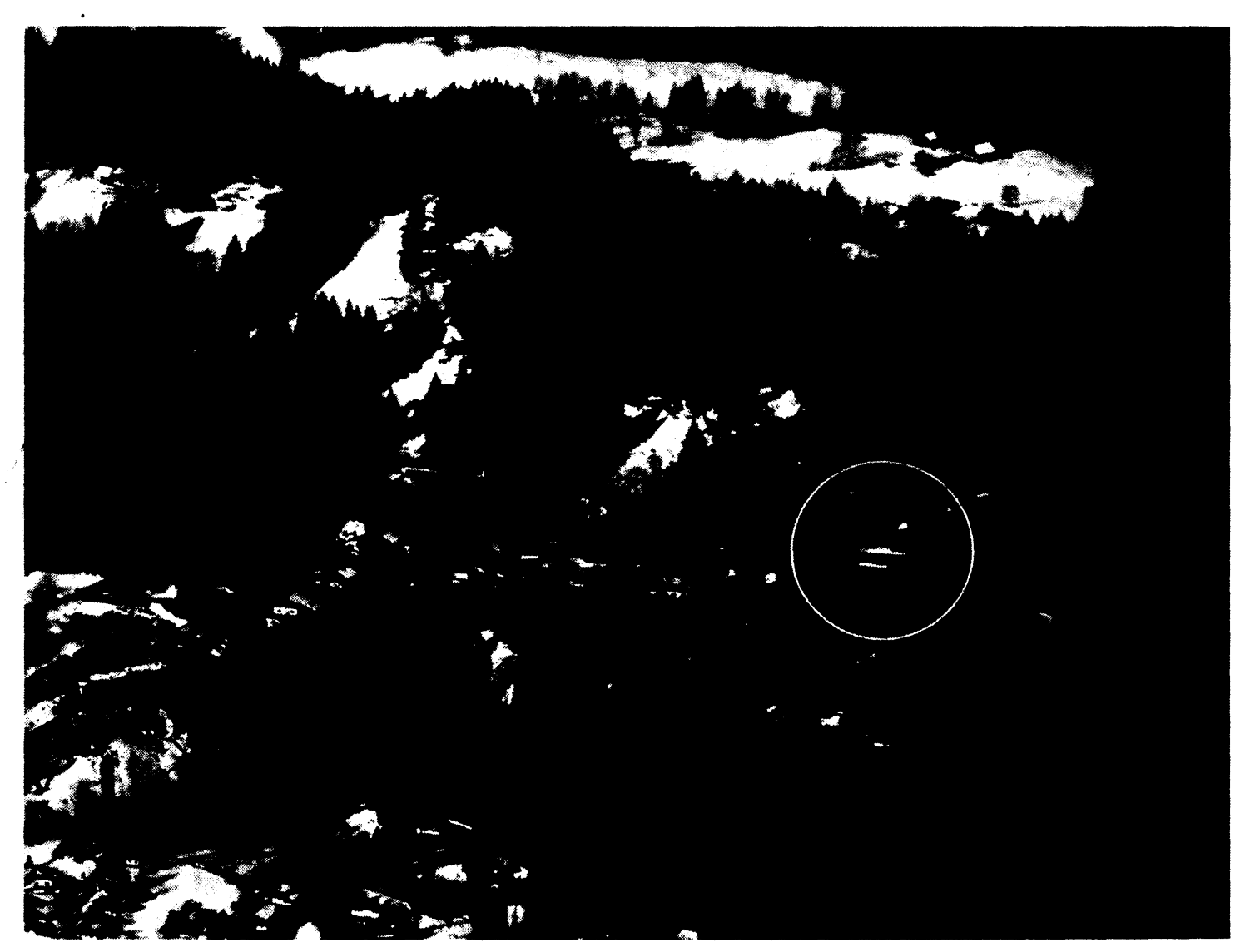

The "Centre de Physique des Houches" (circle) seen from the Aiguille du Midi (alt. $3842 \mathrm{~m}$ ), overlooking Chamonix, some $25 \mathrm{~km}$ away (Photo TM). 